\title{
THE SYDNEY UNIVERSITY STELLAR INTERFEROMETER
}

(SUSI)

\author{
J. DAVIS \\ Chatterton Astronomy Department, School of Physics, University of Sydney
}

\begin{abstract}
.
The Sydney University Stellar Interferometer has been designed and constructed as a two aperture, single $r_{0}$, long baseline, optical interferometer with wavefront-tilt compensation and dynamic optical path length compensation. Initially it will operate in the blue part of the visual spectrum but provision has been made for the addition of an additional beam-combining system for a second spectral band. The rationale behind the choice of instrumental parameters, the potential developments which have been taken into account in its construction, and the current status of SUSI are outlined.
\end{abstract}

Key words: optical - stellar - interferometer

\section{Introduction}

The Sydney University Stellar Interferometer (SUSI) is a long baseline optical amplitude interferometer. In its initial configuration the instrument is a two element interferometer and, because of the random phase differences caused by atmospheric turbulence between the wavefronts received at the two apertures of the instrument, a meaningful visibility phase cannot be measured and images cannot be formed. In order to produce images with separated apertures at optical wavelengths it will be necessary to use the phase-closure techniques developed at radio wavelengths for three or more apertures. To date this has not been achieved although the COAST group at Cambridge are close to testing the technique in the optical regime. Since SUSI is not an imaging instrument at this stage, it would be appropriate to ask why it has been included in an imaging symposium. The reason is straightforward - the problems involved in making a two element optical interferometer work successfully must be overcome before an imaging instrument can be made to work and SUSI includes all the basic features required for each baseline of an imaging instrument.

As other contributors have emphasized, the principles involved in an optical amplitude interferometer are the same as for its radio counterpart. However, optical interferometers have lagged behind their radio counterparts in spite of having had a clear start in the field through the work of Michelson and his colleagues. There are a number of important differences between the optical and radio regimes which are responsible for this lag. The obvious difference is the wavelength which is typically of the order of $10^{5}$ smaller for the optical. This places extremely tight tolerances on the mechanical construction and stability of an optical interferometer although it does have the advantage of giving much higher angular resolution for a given baseline length. A second difference is the need to match the light paths to within a few micrometres before the beams are combined and this generally requires a variable optical path within the instrument which can be controlled at the submicrometre level with extreme smoothness and precision. Atmospheric turbulence distorts the incoming waves of starlight producing randomly varying tilts and phases which must be compensated or accounted for if reliable measurements of fringe 
visibility (visibility amplitude) are to be made. Signal sampling times of a very few milliseconds are necessary to prevent significant phase blurring and this leads to signal levels ranging from a few photons to $\ll 1$ photon per sample period. Thus, photon counting techniques must be employed to measure fringe visibilities.

The problems posed by these differences, which have delayed the development of optical amplitude interferometry, have been solved in SUSI with the aid of modern technology in the form of adaptive optics, laser metrology, photon detectors, computer control, etc.

\section{SUSI}

SUSI is located alongside the Australia Telescope (AT) at the Commonwealth Scientific and Industrial Research Organisation's Paul Wild Observatory, near Narrabri in northern New South Wales, some $500 \mathrm{~km} \mathrm{NNW}$ of Sydney. A number of factors influenced the choice of this site for SUSI. The most important were: (i) the relatively high percentage of clear nights $(\geq 65 \%)$; (ii) the flat nature of the site with sandstone only 2-2.5 m below the surface making suitable foundations and construction straightforward; and (iii) seeing at least as good as at other known potential sites in Australia. Pragmatic reasons included minimal ingoing costs for services to an existing site, the availability of on-site accommodation and the benefits of sharing facilities and expertise with AT staff and observers.

Fig. 1 shows' SUSI's $640 \mathrm{~m}$ long North-South array of siderostat stations. As each station is commissioned it is equipped with a siderostat and relay optics to direct the beam of starlight along an evacuated pipe, which can be seen in the picture, to the central laboratory building. The smaller housings alongside each siderostat station contain the control computer and electronics for the station. They have been located to the west of the baseline, as has the main building, since the prevailing wind direction for fine weather conditions is from the E/SE and this ensures that any hot air generated by the electronics etc. is generally carried away from the baseline. The $80 \mathrm{~m}$ long tunnel-like section of the central building houses an optical path length compensating system (OPLC) (Lawson \& Booth 1993). The basic layout of the instrument has been described by Davis and Tango (1985).

\subsection{SUSI's PARAMETERS}

The design of SUSI is based on a successful prototype instrument (Davis \& Tango 1986). The choice of parameters was driven by our interest in stellar studies and by our experience with the Narrabri Stellar Intensity Interferometer (Hanbury Brown et al. 1974). The range of stellar studies possible with a long baseline optical interferometer has been reviewed by Davis $(1979 ; 1992)$ and McAlister $(1979 ; 1985)$. The choice of parameters was also based on the recognition that, although a two aperture instrument cannot be used to form images, it can contribute a great deal to studies in stellar astrophysics. The need to assume symmetry in the intensity distribution in the absence of the coherence phase is acceptable for many programs as has been discussed by Davis (1979).

Table I lists some of SUSI's parameters. The major parameters characterising 


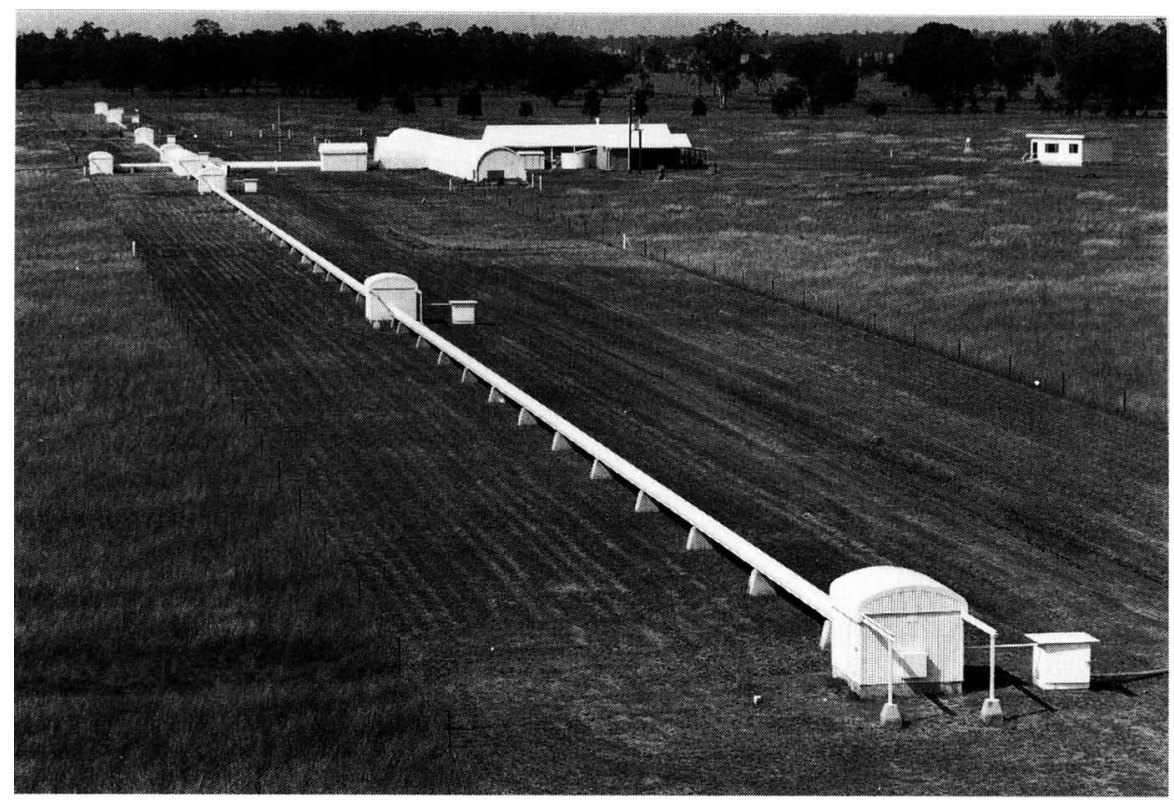

Fig. 1. SUSI seen from the northern end of its $640 \mathrm{~m}$ long North-South baseline array.

the design are the baseline range and configuration, the aperture size and the operating wavelengths.

The range of baselines, namely 5 to $640 \mathrm{~m}$, was chosen to give some overlap with speckle and aperture mask interferometry (e.g. MAPPIT (Bedding et al. 1993)) on the largest optical telescopes for the shortest baselines, and to enable a sample of the hottest $\mathrm{O}$ type stars to be measured with the longest. The sequence of baselines from 5 to $640 \mathrm{~m}$ follows an approximate geometric sequence which ensures that they give an acceptable distribution of spatial frequencies for observations of stars with angular sizes within the resolution range of the instrument. Every second baseline is a factor of two larger with intermediate baselines approximately $\sqrt{ } 2$ greater than the preceding one. The 11 stations on the North-South array give some redundancy in the available baselines but the preferred baselines in the completed array will be $5,10,15,20,30,40,55,80,110,160,220,320,452$ and $640 \mathrm{~m}$ long.

The North-South orientation of the initial baseline array was a pragmatic choice based on the fact that the rate at which the path difference between light from the two apertures changes is much less for a North-South orientation than for an EastWest array - and is zero at transit. It seemed sensible to start from the easier case. The SUSI site is at latitude $30^{\circ}$ South, enabling the maximum sky cover for elevations above $30^{\circ}$ - which was fixed as the minimum elevation for observations with SUSI as the seeing deteriorates rapidly below it. Thus, stars with declinations 
TABLE I

SUSI Parameters

\begin{tabular}{ll}
\hline Baselines: & $5 \mathrm{~m}$ to $640 \mathrm{~m}$ North-South \\
Baseline ratio: & $\sim 1.4$ \\
Angular resolution: & 0.02 to 0.00005 arcseconds \\
Maximum aperture diameter: & $0.14 \mathrm{~m}$ \\
Spectral range: & $400-900 \mathrm{~nm}$ \\
Limiting magnitude: & $\mathrm{V} \sim+8$ \\
\hline
\end{tabular}

from $-90^{\circ}$ to $+30^{\circ}$ can be observed with SUSI. Although the position angle range for a North-South array is poorer than for an East-West array for declinations between $-90^{\circ}$ and $-15^{\circ}$ it is better from $-15^{\circ}$ to $+30^{\circ}$ where the cover for an East-West array is poor - being zero for declination $0^{\circ}$. Ideally, a combination of different baseline orientations is desirable to give a wide range in position angle cover (i.e. $u-v$ plane cover) for the study of asymmetrical objects such as binary stars and rapidly rotating stars. We will return to this point in Section 2.2.

In order to provide the required mechanical stability the siderostats are mounted on massive concrete piers and foundations and are necessarily at fixed locations. The fixed locations mean that wavefronts of starlight arrive at two stations with a path difference which depends on the star's declination and which changes continuously with the Earth's rotation. In order to measure the fringe visibility accurately it is necessary for the beams to be combined with a path difference which is a small fraction of the coherence length of the light. It is therefore necessary to include in the optical paths from the siderostats to the point of beam combination a system to equalise the optical paths. In SUSI this is called the Optical Path Length Compensator (OPLC) system - in other instruments it is called an Optical Delay system or something similar. In principle the path compensation should be done in vacuum since both incoming beams traverse the same optical path through the atmosphere (to first order) and the path difference is essentially a vacuum path difference. For astrometric measurements it is essential to provide the compensating paths in a vacuum but for angular dimensional work it is not necessary. In SUSI the compensating paths are in air for convenience and lower cost. It is important to ensure that turbulence in the instrument, including the compensating air paths, is minimised and this has been achieved by enclosing the internal light paths in a timber enclosure which is inside an air-conditioned volume. The timber enclosure, which is large enough to work in, not only excludes draughts but also provides an additional stage in temperature stabilisation. This arrangement, which was first used in the SUSI prototype, works well. Heat sources have been excluded as far as possible and heat from photomultiplier dynode chains is removed by a circulating water system operating close to ambient temperature. The entry of personnel to the enclosure is minimised prior to observing and everything inside the enclosure is remotely controlled.

Differential dispersion between the two beams will occur with the OPLC in air 
but this can be compensated, as it will be in SUSI, by introducing a combination of glasses of continuously variable thickness into the optical paths so that the dispersion is matched in the two sides by suitable combinations of air, vacuum and glass paths (Tango 1990).

A primary aim in developing SUSI is to obtain accurate measurements of stellar diameters and separations and for this reason it was deemed essential to deal with as much of the deleterious effect of atmospheric turbulence in real time as possible. The approach adopted is to select 'flat' pieces of the incoming wavefront, to correct the wavefront tilts in real time, and to overcome the random phase fluctuations by rapid sampling of the combined beams and on-line processing. The aperture size has therefore been restricted to the order of Fried's atmospheric correlation length, $r_{\mathrm{o}}$, so that essentially flat sections of wavefront are selected by SUSI's apertures. The siderostat mirrors are $200 \mathrm{~mm}$ diameter flats which give a maximum beam diameter of $140 \mathrm{~mm}$ when allowance is made for the projection angle which is always $\leq 45^{\circ}$. This corresponds approximately to $r_{\mathrm{o}}$ for 1 arcsecond seeing at the wavelength of the Balmer $\mathrm{H} \alpha$ line. For shorter wavelengths, and/or poorer seeing, a smaller observing aperture can be selected by rotating wheels containing a range of aperture sizes in the beams. In each arm of the interferometer the tilt of the incoming wavefront is detected and removed by a piezo-electrically mounted mirror thus stabilising the beams along the optical axes of the instrument. It is the wavefront-tilt correcting system that sets the sensitivity limit of the instrument since the corrections have to be made in real time.

Initially the beam-combining optics from the prototype interferometer (Davis \& Tango 1985) are being used in SUSI and these are designed to work in the blue $(\lambda \lambda 400-500 \mathrm{~nm})$. The decision to start with a blue system was made in the early 1980s when photomultipliers were the detector of choice and it was desirable to work near the peak quantum efficiency of the photocathodes. Although there are advantages in using longer wavelengths since the deleterious effects of atmospheric turbulence are wavelength dependent - both $r_{\mathrm{o}}$ and $t_{\mathrm{o}}$ (the correlation time of the phase fluctuations) vary as $\lambda^{6 / 5}$ - the blue end of the spectrum is best for hot stars. Not only is the photon flux higher in the blue but the physical length of baseline needed to resolve the stars is less. As it is, baselines of the order of $600 \mathrm{~m}$ are required at $\lambda 430 \mathrm{~nm}$ to resolve a sample of the hottest $O$ stars.

Initially, the visibility amplitude is being measured using the method described by Tango and Twiss (1980). A PAPA camera is currently being installed to enable fringe tracking to be implemented by means of the channelled spectrum appearing in the dispersed combined beams when the optical paths are not exactly matched (Lawson \& Booth 1993). Shao (1993) discusses detection methods in his paper to this symposium.

A second optical table has been included in the instrument enclosure to carry a beam-combining system to work at the red end of the visual spectrum. It is planned to implement this system in the near future. The spectral range given in Table $I$ is intended only to indicate the range envisaged but it is not limited in principle and the ultimate range will depend on detector availability.

The real time operating system 'which has been developed to control SUSI is described in the poster paper by Minard et al. (1993). 


\subsection{Potential Future Developments}

In the design of SUSI we have kept in mind the possibility of developing a multiaperture imaging capability and have included the first station of an eastern arm in the initial construction. The inclusion of this station makes it possible to add an extended eastern baseline array in the future without disrupting the operation of the North-South baselines. In the first instance such an extension is seen as providing different baseline orientations, by combining the light from an eastern arm station with that from either the southern or northern arms, for improved position angle cover for studies of asymmetrical objects such as rapidly rotating stars, binary stars, etc. This extension would be the necessary first step towards developing a three aperture imaging capability. The inclusion of a fourth beam is possible and the input beam-reducing telescope is designed to handle four beams. The major change, apart from a new beam-combining optical system, would be a re-configuration of the OPLC. The design of the OPLC is such that the re-configuration could be readily achieved.

\section{The Status of SUSI}

SUSI is currently being commissioned, starting with the shorter baselines and with the longer baselines being progressively brought on line. The following list indicates the progress that has been achieved and the current status of the instrument.

- The inner baselines, namely $5,10,15,20,30,40,55$ and $80 \mathrm{~m}$ are currently being commissioned.

- $\quad$ Fringes have been observed with baselines up to and including $80 \mathrm{~m}$ with the OPLC tracking the fringe position.

- Raw correlation or (visibility $)^{2}$ values of $0.56(30 \mathrm{~mm}$ apertures $)$ and 0.68 (18 $\mathrm{mm}$ apertures) for unresolved stars have been obtained at a wavelength of $443 \mathrm{~nm}$.

- $\quad$ Preliminary measurements of Sirius ( $\alpha \mathrm{CMa})$ give an angular diameter in good agreement with the Narrabri Stellar Intensity Interferometer (Hanbury Brown et al. 1974) and SUSI prototype values (Davis \& Tango 1986).

- $\quad$ Seeing has been monitored through 1992 using the wavefront-tilt correction system. The median seeing observed was 1.32 arcseconds with $\leq 1.5$ arcsecond seeing for more than $70 \%$ of the time. This work is summarised in the poster paper by ten Brummelaar et al. (1993).

- It is planned to install the remaining five siderostats of the North-South array during 1993 and to progressively commission the associated baselines from $110 \mathrm{~m}$ to the full $640 \mathrm{~m}$. 


\section{Summary}

The program to commission SUSI is progressing steadily with the inner stations, which give baselines up to $80 \mathrm{~m}$, fully equipped. Fringe visibilities have been measured with baselines up to $80 \mathrm{~m}$ and a program of test observations to identify systematic effects and to calibrate the effects of residual seeing on visibility measurements is being pursued. The siderostats for the longer baselines, from 110-640 m, will be installed with their relay optics during 1993 and progressively brought on line.

Judging from our experience with SUSI to date, we see no insuperable problems in developing long baseline imaging instruments in the visual region of the electromagnetic spectrum.

\section{Acknowledgements}

The design and construction of SUSI has been a team effort and W.J. Tango, A.J. Booth, R.A. Minard, S.M. Owens, and R.R. Shobbrook have shared in the development of the project. Major technical support has come from $\mathrm{H}$. Bennis, M. Paterson, J. Pasiut and J. Hayes. Many postgraduate and honours students have contributed to the project and special mention should be made of T.A. ten Brummelaar, Y.A. Gilliand, J. Giovannis, M. Hrynevych, P.R. Lawson, W.G. Sainty, E.B. Seneta and E.D. Thorvaldson. The Commonwealth Scientific and Industrial Research Organisation (CSIRO) has assisted in many areas including precision optical components, optical coatings, development of the laser metrology system and the leasing of the SUSI site. The construction of SUSI has been funded jointly by the Australian Research Council and the University of Sydney with additional support from the Pollock Memorial Fund and the Science Foundation for Physics within the University of Sydney.

\section{References}

Bedding, T.R., Robertson, J.G. \& Marson, R.G.: 1993, 'Non-redundant masking with MAPPIT at the Anglo-Australian Telescope', In these proceedings.

Davis, J.: 1979, 'The Application of High Angular Resolution Interferometry to the Study of Single Objects in the Visual Region of the Spectrum', in Proc. I.A.U. Colloquium No. 50: High Angular Resolution Interferometry, ed. J. Davis \& W.J. Tango, Chatterton Astronomy Dept., University of Sydney, 1-1 to 1-27

Davis, J.: 1992, 'SUSI's Potential for Binary Star Studies', in Proc. I.A.U. Colloquium No. 135: Complementary Approaches to Double and Multiple Star Research, ed. H.A. McAlister \& W.I. Hartkopf, Astron. Soc. Pacific. Conference Series, 32, 521-526.

Davis, J. \& Tango, W.J.: 1985, 'The Sydney University 11.4 Metre Stellar Interferometer', Proc. A.S.A., 6, 34-38.

Davis, J. \& Tango, W.J.: 1985, 'A New Very High Angular Resolution Stellar Interferometer', Proc.A. S. A., 6, 38-43.

Davis, J. \& Tango, W.J.: 1986, 'New Determination of the Angular Diameter of Sirius', Nature, 323, 234-235.

Hanbury Brown, R., Davis, J. \& Allen, L.R.: 1974, 'The Angular Diameters of 32 Stars', Mon. Not. R. astr. Soc., 167, 121-136.

Lawson, P.R. \& Booth, A.J.: 1993, 'The Fringe Tracking Servo in SUSI', In these proceedings. 
McAlister, H.A.: 1979, 'High Angular Resolution Binary Star Interferometry', in Proc. I.A.U. Colloquium No. 50: High Angular Resolution Interferometry, ed. J. Davis \& W.J. Tango, Chatterton Astronomy Dept., University of Sydney, 3-1 to 3-16.

McAlister, H.A.: 1985, 'High Angular Resolution Measurement of Stellar Properties' in Ann. Rev. Astron. Astrophys., 23, 59-87.

Minard, R.A., Booth, A.J., Tango, W.J. \& ten Brummelaar, T.: 1993, 'An Overview of the SUSI Control System', In these proceedings.

Shao, M.: 1993,'Fringe Visibility and Phase Measurements', In these proceedings.

Tango, W.J.: 1990, 'Dispersion in Stellar Interferometry', Applied Optics, 29, 516-521.

Tango, W.J. and Twiss, R.Q.: 1980, 'Michelson Stellar Interferometry', Progress in Optics, XVII, 241-277.

ten Brummelaar, T., Tango, W.J., Davis, J. \& Shobbrook, R.R.: 1993, 'Preliminary Seeing Measurements with SUSI', In these proceedings.

\section{Discussion:}

Baldwin:

Could you say something about the bandwidths used and the considerations determining the choice of bandwidth?

Davis:

For the commissioning tests we have generally used a bandwidth of $0.75 \mathrm{~nm}$. Because of the corresponding large coherence length $(\sim 260 \mu \mathrm{m}$ at $\lambda 442 \mathrm{~nm})$ it is relatively easy to locate the fringes when the baseline parameters are uncertain. As the baseline solutions improve wider bandwidths become practical, the limitation being set by differential dispersion in the differing airpaths in SUSI. This will be compensated using a combination of glass, air and vacuum paths as discussed above. 